\title{
Development
}

\section{Pannexin 1 Regulates Dendritic Protrusion Dynamics in Immature Cortical Neurons}

\author{
DJuan C. Sanchez-Arias, Rebecca C. Candlish, Emma van der Slagt, and 는 Aigh Anne Swayne
}

https://doi.org/10.1523/ENEURO.0079-20.2020

Division of Medical Sciences, University of Victoria, Victoria, British Columbia V8P 5C2, Canada

\begin{abstract}
The integration of neurons into networks relies on the formation of dendritic spines. These specialized structures arise from dynamic filopodia-like dendritic protrusions. It was recently reported that cortical neurons lacking the channel protein pannexin 1 (PANX1) exhibited higher dendritic spine densities. Here, we expanded on those findings to investigate, at an earlier developmental time point (with more abundant dendritic protrusions), whether differences in the properties of dendritic protrusion dynamics could contribute to this previously discovered phenomenon. Using a fluorescent membrane tag (mCherry-CD9-10) to visualize dendritic protrusions in developing neurons [at $10 \mathrm{~d}$ in vitro (DIV10)], we confirmed that lack of PANX1 led to higher protrusion density, while transient transfection of Panx1 led to decreased protrusion density. To quantify the impact of PANX1 expression on protrusion formation, elimination, and motility, we used live cell imaging in DIV10 neurons (one frame every $5 \mathrm{~s}$ for $10 \mathrm{~min}$ ). We discovered that at DIV10, loss of PANX1 stabilized protrusions. Notably, re-expression of PANX1 in Panx1 knock-out (KO) neurons resulted in a significant increase in protrusion motility and turnover. In summary, these new data revealed that PANX1 could regulate the development of dendritic spines, in part, by controlling dendritic protrusion dynamics.
\end{abstract}

Key words: cortical neuron; dendritic protrusions; dendritic spines; live imaging; neurodevelopment; pannexins

\section{Significance Statement}

Dendritic spines are microscopic structures that allow for communication between brain cells. Previous work showed that pannexin 1 (Panx1) knock-out (KO) increases the density of dendritic spines, raising the possibility that PANX1 could regulate their formation and/or stability. To address this research question, here we studied the role of Panx $1 \mathrm{KO}$ and rescue on dendritic protrusions, the dynamic precursors of dendritic spines, in immature developing neurons. We found that Panx1 KO increased the density and stability of protrusions on developing neurons, and conversely, that PANX1-EGFP expression decreased protrusion density, and increased protrusion turnover and overall movement. These results enhance our understanding of PANX1 regulation of neuronal development and neuroplasticity.

\section{Introduction}

Although the mechanisms controlling plasticity of established dendritic spines are relatively well-characterized (Holtmaat et al., 2005; Araya et al., 2014; Sala and Segal, 2014; Schätzle et al., 2018), the molecular processes underlying their formation are less clear (Sando et al., 2017; Sigler et al., 2017; for review, see Südhof, 2018). Pannexin 1 (PANX1) is a four transmembrane domain

Received March 3, 2020; accepted July 21, 2020; First published July 31, 2020.

The authors declare no competing financial interests. protein that forms channels permeable to ions and metabolites with various activation mechanisms and diverse (patho)physiological implications (for review, see Boyce et al., 2018; Chiu et al., 2018). PANX1 is broadly and highly expressed in the brain during early postnatal development (Ray et al., 2005; Vogt et al., 2005) and localized and enriched in synaptic compartments (Zoidl et al., 2007; Sanchez-Arias et al., 2019). Pharmacological and genetic

Author contributions: J.C.S.-A. and L.A.S. designed research; J.C.S.-A. and R.C.C. performed research; J.C.S.-A., E.v.d.S., and L.A.S. analyzed data; All authors wrote and revised the paper. 
disruption of PANX1 results in enhanced induction of longterm potentiation (LTP) and impaired induction of longterm depression (LTD) in the hippocampus (Prochnow et al., 2012; Ardiles et al., 2014; Gajardo et al., 2018). Given that PANX1-interacting cytoskeletal regulators, collapsin response mediator protein 2 (CRMP2) and actin-related protein 2/3 complex (ARP2/3), are known to control dendritic spine formation (Wicki-Stordeur and Swayne, 2013; Spence et al., 2016; Zhang et al., 2016; Xu et al., 2018), it was perhaps not surprising that Panx 1 deletion led to the formation of larger cortical neuron network ensembles and that this was due, in part, to increased cortical neuron dendritic spine density (Sanchez-Arias et al., 2019).

It was unclear from the previous study (Sanchez-Arias et al., 2019) whether the increase in dendritic spine density associated with Panx1 knock-out (KO) was because of an increase in the formation and/or an increase in the stability of the processes that develop into dendritic spines. Moreover, the previous study did not address whether re-expression of PANX1 could "rescue" the impact of Panx1 KO. Dendritic spines develop from long, thin, highly dynamic processes called "dendritic protrusions" that are motile (i.e., grow and retract), and either disappear relatively rapidly (on the order of tens of seconds to minutes) or "survive" and stabilize. The goals of the current study were therefore 2-fold: (1) to investigate the impact of PANX1 on dendritic protrusion density and dynamics in developing cortical neurons; and (2) to determine whether the impact of Panx1 KO could be rescued by re-expression of PANX1.

Because dendritic protrusion development is more readily accessible to study in vitro, and these systems (e.g., primary neuronal cultures) recapitulate many features of spine development observed in vivo (Ziv and Smith, 1996; Dunaevsky et al., 1999; Prange and Murphy, 2001; Zuo et al., 2005), we established an approach to study dendritic protrusions in immature cortical neuron

This work was supported by operating grants from the Canadian Institutes of Health Research (MOP142215), the Natural Sciences and Engineering Research Council of Canada (RGPIN-2017-03889), The Scottish Rite Charitable Foundation of Canada (15118), and the University of Victoria-Division of Medical Sciences (to L.A.S.). L.A.S. was also supported by a Michael Smith Foundation for Health Research and British Columbia Schizophrenia Society Foundation Scholar Award (5900). J.C.S.-A. was supported by a University of Victoria Fellowship Graduate Award. E.v.d.S. was supported by a Natural Sciences and Engineering Research Council of Canada Undergraduate Student Research Award. Infrastructure support was given by the Canada Foundation for Innovation (29462), and the BC Knowledge Development Fund (804754) supported the Leica SP8 microscope system.

Acknowledgements: We thank Sarah N. Ebert who was supported by a Jamie Cassels Undergraduate Research Award for technical assistance. We also thank Reg Sidhu (Leica Microsystems) for assistance in the optimization of our live imaging set up. This article was previously posted as a preprint on bioRxiv (https://doi.org/10.1101/2020.03.02.973917). Note that no additional experiments were completed after the initial submission due to the COVID-19 pandemic; these data are also included in the PhD thesis of J.C.S.-A (Sanchez-Arias, 2020)

Correspondence should be addressed to Leigh Anne Swayne at Iswayne@uvic.ca.

https://doi.org/10.1523/ENEURO.0079-20.2020

Copyright (C) 2020 Sanchez-Arias et al.

This is an open-access article distributed under the terms of the Creative Commons Attribution 4.0 International license, which permits unrestricted use, distribution and reproduction in any medium provided that the original work is properly attributed. cultures. Dendritic protrusions are long, thin, and contain relatively little cytoplasm. Therefore, standard cytoplasmic fluorescent proteins are not ideal for studying dendritic protrusions in immature, developing neurons. To this end, we devised a novel approach using the transmembrane tetra-spanin CD9-10 (Tspan29) protein fused to the monomeric red fluorescent protein mCherry (mCherry-CD9-10) and imaged dendritic protrusion dynamics in living neurons over a 10-min time interval. To determine the impact of Panx1 KO on dendritic protrusion density and dynamics, and also to determine whether any observed effects could be rescued by re-expression of PANX1, we transiently transfected wild-type (WT) and Panx1 KO neuronal cultures with control EGFP or PANX1-EGFP (as well as mCherry-CD9-10) and analyzed dendritic protrusions in fixed and living neurons at $10 \mathrm{~d}$ in vitro (DIV10). We confirmed that loss of PANX1 led to higher dendritic protrusion density. As anticipated, rescue of PANX1 in Panx1 KO cultures, and transient expression of PANX1 in WT cultures led to decreased dendritic protrusion densities. Moreover, Panx1 KO was associated with increased dendritic protrusion stability. Finally, transient PANX1 expression in Panx1 KO cultures significantly increased motility and turnover of dendritic protrusions. In summary, these new data revealed an inverse relationship between PANX1 expression levels and dendritic protrusion stability, suggesting that the loss of PANX1, either through genetic deletion or during developmental downregulation, results in increased cortical dendritic spine density, in part by stabilizing dendritic protrusions.

\section{Materials and Methods}

\section{Experimental animals}

All animal procedures were approved by the University of Victoria Animal Care Committee and performed in accordance with the guidelines set by the Canadian Council on Animal Care. Male and female postnatal day (P)0-P1 mice were used in this study. C57BL/6J mice were obtained from The Jackson Laboratory (\#000664, Table 1). The global Panx $1 \mathrm{KO}$ strain in this study was derived from the Panx $1^{\text {Vsh }}$ null mice, originally generated by Valery Shestopalov (Dvoriantchikova et al., 2012). Note that this strain was developed using targeted mutagenesis on 129embryonic stem cells (129-ESCs, $129 \times 1 /$ SvJ x 129S1/Sv) with $\mathrm{C} 57 \mathrm{BL} / 6 \mathrm{~J}$ as the recipient strain and backcrossed for five generations by the original authors. Recent comparative genomic analysis revealed the original Pan $x 1^{\mathrm{Vsh}}$ null mice contained a loss of function passenger mutation in Casp4 (Casp11), common to five 129-substrains $(129 \times 1 /$ SvJ, 129S1/SvImJ, 129S2/SvPas, 129S6/SvEvTac and 129P3/J) and are predicted to have passenger mutations in these additional genes: Mmp1a, Olfr832, Fbxl12, ENSMUSG00000095186, and ENSMUSG00000095891 (Vanden Berghe et al., 2015). These mice were further backcrossed in-house onto a C57BL/6J for at least six generations (Sanchez-Arias et al., 2019). Mice were housed under a 12/12 h light/dark cycle starting at $8 \mathrm{~A}$. M., with food and water ad libitum; temperature was maintained between $20^{\circ} \mathrm{C}$ and $25^{\circ} \mathrm{C}$ and humidity at $40-65 \%$. 
Table 1: Key resources table

\begin{tabular}{|c|c|c|c|}
\hline Reagent or resource & Source & Identifier & RRID \\
\hline \multicolumn{4}{|l|}{ Experimental models: organisms/strains } \\
\hline $\begin{array}{l}\text { Cortical neuron cultures from P0 Panx1 } \\
\text { KO on a C57BL/6J background }\end{array}$ & $\begin{array}{r}\text { (Dvoriantchikova et al., 2012; } \\
\text { Sanchez-Arias et al.. 2019) }\end{array}$ & NA & NA \\
\hline \multicolumn{4}{|l|}{ Recombinant DNA } \\
\hline mCherry-CD9-10 & Addgene & Plasmid \#55013 & RRID:Addgene_55013 \\
\hline pEGFP-N1 & Clontech (Takara Bio), discontinued & Catalog \#6085-1 & NA \\
\hline DMEM/F12 & Thermo Fisher Scientific & & NA \\
\hline NeuroCult & STEMCELL Tech. & Catalog \#05713 & NA \\
\hline BrainPhys & STEMCELL Tech. & Catalog \#05790 & NA \\
\hline Neurocult SM1 & STEMCELL Tech. & Catalog \#05711 & NA \\
\hline GlutaMAX & Thermo Fisher Scientific & Catalog \#35050061 & NA \\
\hline $\mathrm{P} / \mathrm{S}$ & Thermo Fisher Scientific & Catalog \#15140122 & NA \\
\hline DNAse-1 & MilliporeSigma & 11284932001 & NA \\
\hline Cytosine $\beta$-D-arabinofuranoside (ara-C) & MilliporeSigma & C1768 & NA \\
\hline Lipofectamine2000 & Thermo Fisher Scientific & Catalog \#11668027 & NA \\
\hline OptiMEM I & Thermo Fisher Scientific & Catalog \#31985062 & NA \\
\hline Probenecid (water-soluble) & Thermo Fisher Scientific & Catalog \#P36400 & NA \\
\hline My-Taq Extract PCR kit & Bioline & $\mathrm{BIO}-21126$ & NA \\
\hline Vectashield & Vector Laboratories & $\mathrm{H}-1000$ & RRID:AB_2336789 \\
\hline \multicolumn{4}{|l|}{ Software and algorithms } \\
\hline FIJI (FIJI is just ImageJ) & NIH (Schindelin et al., 2012) & NA & RRID:SCR_002285 \\
\hline MultiStackReg v1.45 & Brad Busse (http://bradbusse.net/ & NA & NA \\
\hline $\begin{array}{l}\text { R Project for Statistical Computing } \\
\text { (version 3.6.2) }\end{array}$ & $\begin{array}{l}\text { MultiStackReg1.45_.jar) } \\
\text { The R Foundation }\end{array}$ & NA & RRID:SCR_001905 \\
\hline Rstudio & Rstudio Inc. & NA & RRID:SCR_000432 \\
\hline 8-well Nunc Lab-Tek Chamberedcoverglass & Thermo Fisher Scientific & 155411PKGG- & NA \\
\hline PDL precoated coverslips & NeuVitro & 12-PDL & NA \\
\hline
\end{tabular}

\section{Primary cortical neuron cultures and transfections}

Primary cortical neuron cultures were prepared as previously described (Sanchez-Arias et al., 2019). Briefly, cortices from male and female P0 pups from timed-pregnant WT and Panx1 KO breeding pairs were microdissected and incubated with papain, dispase-1, and DNase1 for 40 min in HBSS, followed by mechanical dissociation in DMEM/F12 medium supplemented with Neurocult SM1, GlutaMAX, and penicillin/streptomycin (P/S). A total of 125,000 cells were plated in a Nun Lab-Tek eight-well chambered coverglasses coated with PDL. One to two hours after plating, the medium was replaced with Neurocult supplemented with Neurocult SM1, GlutaMAX, $\mathrm{P} / \mathrm{S}$, and gentamicin. From DIV4 onwards, partial (half) medium changes were done with BrainPhys maturation medium (Bardy et al., 2015); to limit proliferation of glial cells, ara-C was added to the medium at DIV4. Transfections were performed at DIV6 using Lipofectamine2000. DNA/ lipid complexes were diluted in OptiMEM-I at a ratio of $2 \mu \mathrm{g}$ DNA: $1 \mu$ lipofectamine and incubated at room temperature for $30 \mathrm{~min}$. Then, these DNA/lipid complexes were added to cells in BrainPhys medium without antibiotics and incubated for $1-1.5 \mathrm{~h}$. Neurons were transfected with either pEGFP-N1 (250 ng) or Panx1-EGFP (250 ng, gift from Silvia Penuela and Dale Laird; Penuela et al., 2007). All transfections contained mCherry-CD9-10 (250 ng, a gift from Michael Davidson; Addgene plasmid \#55013; http://n2t. net/addgene:55013; RRID:Addgene_55013) to visualize neurons and dendritic protrusions. All neurons in this study were used at DIV10. Neurons used for fixed quantifications were plated on PDL-coated coverslips.

\section{Genotyping}

A set of primers targeting upstream exon 3 , exon 3 , and downstream exon 4 of Panx1 (CTTTGGCATTTTCCCA GTGT, CGCGGTTGTAGACTTTGTCA, and GTCCCTACA 
GGAGGCACTGA) were used to genotype mice as previously described (Sanchez-Arias et al., 2019). Genomic DNA was extracted from tail-clips using MyTaq Extract PCR kit. DNA from WT mice amplifies a single 585-bp band, whereas DNA amplified from global Panx1 KO mice have a single 900-bp band.

\section{Imaging and analysis of dendritic protrusions in fixed cortical neurons}

Dendritic protrusions (including filopodia) were defined as any membranous protrusions between 0.4 and $10 \mu \mathrm{m}$. Neurons were fixed on coverslips with $4 \%$ paraformaldehyde and $4 \%$ sucrose for $10 \mathrm{~min}$ and mounted on microscope slides with VectaShield antifade mounting medium. High-resolution images $(3320 \times 3320$, pixel size: $0.088 \mu \mathrm{m}$, $z$-step size: $0.4 \mu \mathrm{m})$ were acquired using a Leica TSC SP8 microscope using a $40 \times$ immersion oil objective (1.30 NA) and exported to FIJl for analysis (Schindelin et al., 2012). Two experimenters were involved in dendritic protrusion density analysis. One experimenter quantified dendritic protrusions according to the inclusion criteria [neurons that expressed both mCherry-CD9-10 and EGFP/PANX1-EGFP, did not show signs of stress (blebbing, large vacuoles), and where dendritic protrusions were originating from the longest primary neurite] from all experimental groups. The other experimenter quantified dendritic protrusions only from mCherryCD9-10 and EGFP-expressing neurons to obtain quantitative analysis of the improvement in dendritic protrusion detection realized with mCherry-CD9-10 versus EGFP signal and record interobserver variance in protrusion analysis. For quantitative assessment of dendritic protrusion detection realized with mCherry-CD9-10 versus EGFP signal, neurons were randomly selected by a second experimenter for analysis. Using the mCherry-CD9-10 signal, the experimenter traced individual dendritic protrusions emanating from the shaft of the longest neurite (primary neurite). The corresponding EGFP signal for each dendritic protrusion (initially traced using the mCherry-CD9-10 signal) was inspected and the presence or absence of a corresponding dendritic protrusion in the EGFP channel was recorded. The interobserver variability was determined by Pearson's correlation and reported as $R^{2}$. Dendritic protrusions from all experimental groups were traced in the same manner, and densities were calculated by dividing the total number of dendritic protrusions by the segment length and multiplying by 10 (dendritic protrusions per $10 \mu \mathrm{m})$. Representative images were processed uniformly with a Gaussian blur of 0.5 pixels, and uniform adjustments to levels and contrast were made using Photoshop CS6 Extended suite (Adobe Systems).

\section{Imaging and analysis of dendritic protrusions in live cortical neurons}

Cortical neurons plated on chambered coverglasses and cultured in BrainPhys medium were placed in an incubation chamber attached to a Leica TSC SP8 microscope and held at $37^{\circ} \mathrm{C}$ and $5 \% \mathrm{CO}_{2}$ for the entire duration of the experiment. After $15 \mathrm{~min}$ of acclimatization, primary and secondary dendrite segments within $100-150 \mu \mathrm{m}$ from the cell body were imaged. We selected segments from the longest neurite (dendrite) that were clearly discernible from surrounding structures (other dendrites and cells). These segments ranged from $\sim 65$ to $\sim 75 \mu \mathrm{m}$ long. Time lapses of $z$-stacks $(0.7-\mu \mathrm{m} z$-step size) were acquired every $5 \mathrm{~s}$ for $10 \mathrm{~min}$ at a resolution of $1024 \times 256$ (pixel size: $0.06 \mu \mathrm{m}$ ) using Leica's TCS SP8 resonant mode $(8000 \mathrm{~Hz})$ and a $63 \times$ water immersion objective (1.20 NA). Images were exported to FIJI for analysis. First, the four-dimensionality $(x, y, z, t)$ was reduced by creating maximum $z$ projections before additional image processing, $x-y$ drift was corrected with MultiStackReg v1.45 (developed by Brad Busse; http://bradbusse.net/ MultiStackReg1.45_.jar) when required. Then, images were subjected to a low-pass filter using a Gaussian blur (kernel size 2) and thresholded using the triangle method (Zack et al., 1977; Polanco et al., 2018). From these binary images, outlines for each time frame were created and temporally color coded. Dendritic protrusions were manually counted, and four basic characteristics were recorded: formation, elimination, lability, and motility. We defined formation as any de novo appearance of a dendritic protrusion within the timelapse recording; elimination was defined as the complete disappearance of a dendritic protrusion. Lability was defined as dendritic protrusions that were formed and eliminated within the duration of the time lapse, typically short-lived and lasting 1-3 min. To assess dendritic protrusion motility, we annotated partial extensions and partial retractions of individual dendritic protrusions. The survival fraction of dendritic protrusions was calculated by dividing the number of dendritic protrusions at the end of each time lapse (10-min mark) by the number of dendritic protrusions at the start (0-min mark). The overall turnover rate was calculated as the net percent gain and loss (sum of formation, elimination, and lability) of dendritic protrusions divided by the number of dendritic protrusions at the start of the time lapse. Lastly, the overall movement change of dendritic protrusions ( $\Delta$ movement) was calculated by adding the fundamental characteristics of dynamics (formation, elimination, lability, and motility) divided by the number of dendritic protrusions at $0 \mathrm{~min}$. Neurons that displayed signs of blebbing or for which focal planes were lost during the acquisition of images were excluded from analysis. Representative images were processed uniformly with a Gaussian blur of 0.5 pixels, and uniform adjustments to levels and contrast were made using Photoshop CS6 Extended suite (Adobe Systems Inc.).

\section{Experimental design and statistical analysis}

Note that no additional experiments were completed after the initial submission because of the COVID-19 pandemic; these data are also included in the $\mathrm{PhD}$ thesis of J.C.S.-A (Sanchez-Arias, 2020). For experiments with fixed neurons, two coverslips from three independent cultures were used; for live cell imaging experiments, two to three eight-well chambered coverglasses from three independent cultures were used. Neurons from either WT or Panx1 KO mice were plated in PDLcoated eight-well chambered cover glass or on PDLcoated coverslips in a randomized order by one 
A
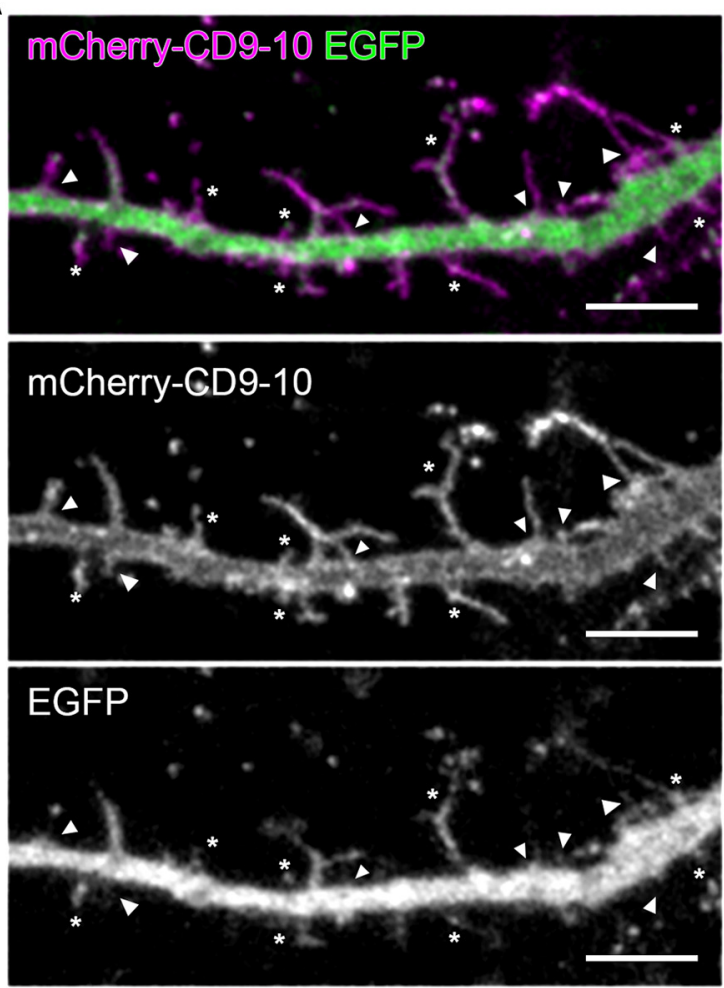

B

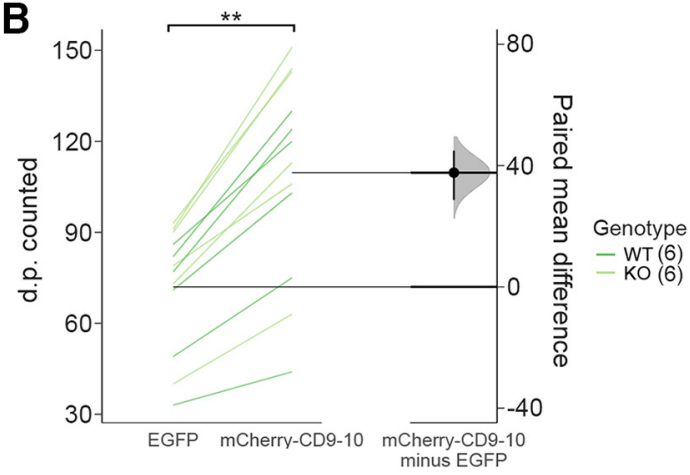

C

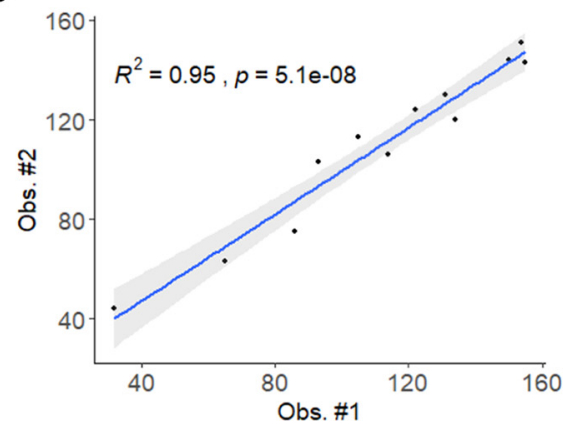

Figure 1. Detection of dendritic protrusions in cortical neurons was improved with a membrane-bound fluorescent marker. $\boldsymbol{A}$, Representative maximum intensity projection of a dendritic segment from a neuron transfected with mCherry-CD9-10 and EGFP at DIV6 and fixed at DIV10. Thin and long dendritic protrusions are more clearly visualized with mCherry-CD9-10 (mid) than the cytoplasmic volume marker EGFP (bottom). Structures not clearly labeled with EGFP are denoted by $*$, and those missed entirely are denoted with arrowheads. B, Slopegraph showing the quantification of dendritic protrusions detected using the mCherry-CD9-10 signal compared with the EGFP signal. On average, 34\% of dendritic protrusions detected with mCherry-CD9-10 were missed in the EGFP channel (EGFP: $72 \pm 5.9$ dendritic protrusion; mCherry-CD9-10: $110 \pm 9.7$ dendritic protrusions; $p=0.00384$, Student's $t$ test; $N=12$ neurons, 6 WT and 6 Panx1 $\left.\mathrm{KO}^{\mathrm{a} 1}\right)$. C, The interobserver variability was evaluated with Pearson's correlation and found to be $R^{2}=0.95(95 \mathrm{Cl} 0.84-0.98$, $\left.p=5.1 \mathrm{e}^{-08}\right)^{\mathrm{a} 2}$. d.p., dendritic protrusion; $* *<0.01$. Effect sizes are reported in Table 2. Scale bar: $5 \mu \mathrm{m}$. These data are included in the $\mathrm{PhD}$ thesis of J.C.S.-A, University of Victoria (Sanchez-Arias, 2020), found at http://hdl.handle.net/1828/11714.

experimenter, and all transfections, imaging, and image analysis were performed without knowledge of the genotype of the cultures by another experimenter. Neurons that displayed signs of blebbing or for which the focal plane was lost during image acquisition were excluded from analysis. Relevant details are described in Results, figure legends, and where appropriate, illustrated on the figures themselves. Data are presented as mean \pm SD. Data analysis using bootstrap estimation (5000 bootstrap resamples), determination of effect size, bias-corrected confidence intervals, and Cumming estimation plots were generated using the dabestr package for $\mathrm{R}$ (Bernard, 2019; Calin-Jageman and Cumming, 2019; Ho et al., 2019). Null-hypothesis significance testing was performed using $\mathrm{R}$ (version 3.6.2), and $p<0.05$ was used as the significant threshold for these tests. Normality was tested using the Shapiro-Wilk test (McDonald, 2014). Group analyses for normally distributed data were performed with a two-way ANOVA coupled to multiple comparisons with Bonferroni's correction. For non-normally distributed data, KruskalWallis pairwise comparisons with Bonferroni's correction were used.

\section{Results}

\section{Detection of dendritic protrusions in cortical neurons was improved with a membrane-bound fluorescent marker}

Dendritic spines develop from highly dynamic dendritic protrusions. Genetic methods used to detect and measure these structures have mainly consisted of expression of cytoplasmic fluorescent proteins, such as EGFP. Transient transfection of EGFP (or one of its many variants) represent a convenient way to sparsely label neurons. There is a reasonable concern, however, that these cytoplasmic markers could fail to detect a substantial proportion of dendritic protrusions, given that these structures are relatively thin (and therefore contain a relatively small amount of cytoplasm; Fig. 1A). Membrane-bound lipophilic dyes (Dil, DiO, etc.), on the other hand, label thin membrane processes quite well (Mancuso et al., 2013). In order to combine the convenience of transient transfection of fluorescent proteins with the effectiveness of membranebound labels in thin process detection, we transfected cortical neurons with a fluorescent protein, mCherry, fused to a transmembrane protein, tetraspanin CD9-10 (mCherry-CD9- 
10, from Michael Davidson, Addgene plasmid \#55013). As expected, the fluorescence signal from mCherry-CD9-10 delineated the plasma membrane (in addition to detecting somatic puncta most likely representing endosomes; Polanco et al., 2018). Qualitatively, it appeared that mCherry-CD9-10 improved the detection of dendritic protrusions. To quantify the improvement with mCherry-CD9-10, we traced dendritic protrusions using the mCherry-CD9-10 signal and compared this with the EGFP signal to confirm whether or not the same dendritic protrusions could be detected in that channel. We found that $\sim 34 \%{ }^{\mathrm{a} 1}$ of dendritic protrusions detected using the mCherry-CD9-10 signal were not readily detected with the EGFP signal (Fig. 1B). We compared the number of dendritic protrusions detected between two observers using Pearson's correlation and found them to be $R^{2}=0.95^{22}$, suggesting mCherry-CD9-10 facilitated dendritic protrusion detection independent of the observer (Fig. 1C). These results suggest that mCherryCD9-10 represents an improved method for measuring dendritic protrusions in neurons.

\section{PANX1-EGFP expression rescued the increase in} dendritic protrusion density associated with Panx1 KO

To investigate the impact of transient PANX1 expression on dendritic protrusion density in immature neurons, we transfected DIV6 WT and Panx1 KO cortical neuronal cultures with mCherry-CD9-10 as well as EGFP (control) or PANX1-EGFP (overexpression for WT cultures, rescue for KO cultures). We fixed the cells $4 \mathrm{~d}$ later at DIV10 and measured the density of dendritic protrusions (Fig. 2A). In EGFP control-expressing cultures, we observed a $20 \%$ increase in dendritic protrusion density in primary neurites of Panx1 KO neurons (effect size: 2.36 [95\% confidence interval $\left.(95 \mathrm{Cl}) 1.28 ; 3.54], p=0.03517,{ }^{\mathrm{b} 1}\right)$ compared with WT controls. In PANX1-EGFP-expressing cultures, we observed a $27 \%$ decrease in dendritic protrusion density in WT neurons (effect size: -3.24 [95Cl $-4.54 ;-2.21]$, $p=0.00268^{\text {b1 }}$; Fig. $2 B i$ ) and a $42.5 \%$ density reduction in Panx1 KO neurons (effect size: -6.08 [95Cl -7.84; -4.51], $p<0.0001^{\mathrm{b} 1}$; Fig. 2Bi) compared with EGFP-expressing WT controls. Dendritic protrusion length was not significantly different among the groups (Fig. 2Biib2). These results suggested dendritic protrusion density was inversely proportional to PANX1 expression levels.

\section{Novel methods for measurement of dendritic protrusions dynamics in living neurons}

To examine the mechanisms contributing to differences in dendritic protrusion densities between groups, we acquired 10-min time lapses (one frame every $5 \mathrm{~s}$ ) of primary and secondary dendrites from cortical neurons at DIV10. These cultures were transfected with mCherry-CD9-10 and either EGFP or Panx1-EGFP at DIV6. At DIV10, dendrites harbor highly dynamic, thin, and long dendritic protrusions that are the precursors for dendritic spines (Ziv and Smith, 1996; Fiala et al., 1998). We reduced the dimensionality of the time lapses by creating maximum $z-$ projections, and then images were passed through a lowpass filter and thresholded to create outlines (Fig. 3A). The dendritic silhouettes (Fig. $3 B$ ) were then temporally color coded to facilitate the detection of formation, elimination, lability, retraction, and growth of dendritic protrusions (Fig. 3C).

\section{Metrics of dendritic protrusion dynamics correlated with PANX1 expression levels}

Using our newly developed approach for the measurement of dendritic protrusion dynamics, we found that transient PANX1-EGFP expression significantly increased the formation (de novo appearance) and elimination (complete disappearance) of dendritic protrusions in Panx $1 \mathrm{KO}$ neurons compared with transient EGFP expression (formation: effect size KO-EGFP vs KO-PANX1-EGFP: $8.23 \%$ [95Cl 4.54\%; $11.8 \%$ ], $p=0.0028^{\mathrm{c} 1}$; elimination: effect size KO-EGFP vs KO-PANX1-EGFP: $11.6 \%$ [95Cl $7.5 \%$; $15.8 \%$ ], $p=0.00024^{\mathrm{c} 2}$; Fig. $\left.4 A, B i, B i i\right)$, while no significant differences were observed between PANX1EGFP and EGFP expressing WT cultures $\left(p>0.9999^{c 1}\right)$. Similarly, no significant differences were observed between genotypes in EGFP control-expressing cultures (formation: effect size WT-EGFP vs KO-EGFP: $-3.02 \%$ $[95 \mathrm{Cl}-5.39 \% ;-0.803 \%], p=0.2267^{\mathrm{c} 1}$; elimination: effect size WT-EGFP vs KO-EGFP: $-2.57 \%[95 \mathrm{Cl}-5.85 \%$; $-0.209 \%$ ], $p=0.62307^{\mathrm{c} 2}$; Fig. $\left.4 \mathrm{Bi}, \mathrm{Bii}\right)$. We next quantified protrusion lability, a term we assigned to dendritic protrusions that transiently appeared and disappeared within 1-3 min (i.e., during our analysis period). Transient PANX1EGFP expression significantly increased dendritic protrusion lability in Panx1 KO neurons; whereas, there was significant impact of PANX1-EGFP expression in WT neurons (effect size KO-EGFP vs KO-PANX1-EGFP: 7.31\% [95Cl 3.92\%; $10.6 \%$ ], $p=0.0034$; effect size WT-EGFP vs WT-PANX1EGFP: $2.34 \%$ [95Cl $-0.966 \%$; $6.76 \%], p>0.9999^{\mathrm{c} 3}$; Fig. 4Biii). WT and Panx1 KO EGFP-expressing cultures exhibited no differences in dendritic protrusion lability (effect size WT-EGFP vs KO-EGFP: $-1.11 \%$ [95Cl $-3.17 \%$; $0.786 \%]$, $p>0.9999^{\mathrm{c}}$ ). As anticipated, Panx1 KO neurons exhibited significantly reduced dendritic protrusion motility (partial extension or retraction of an existing dendritic protrusion) compared with WT neurons within EGFP control-expressing cultures (effect size WT-EGFP vs KO-EGFP: $-13.3 \%$ [95Cl $-18.5 \%$; $-8.42 \%$ ], $p=0.00016^{\mathrm{c} 4}$; Fig. 4Biv). Intriguingly, transient PANX1-EGFP expression increased dendritic protrusion motility in Panx1 KO neurons only (effect size KOEGFP vs KO-PANX1-EGFP: $10.2 \%$ [95Cl $4.53 \%$; $16 \%$ ], $\left.p=0.03582^{c 4}\right)$. Together, these results suggest that dendritic protrusion movement roughly correlated with PANX1 expression levels.

\section{PANX1 increased dendritic protrusion turnover and overall movement}

To determine the overall impact of PANX1 expression on dendritic protrusion stability, we next used the fundamental metrics devised in Figure $3 C$ to calculate dendritic protrusion survival, turnover, and overall change in movement ( $\Delta$ movement). Survival, or the percentage of dendritic protrusions persisting at the end of the analysis period relative to time $0 \mathrm{~min}$, was significantly reduced in PANX1-EGFP expressing neurons compared with EGFP control- 
A
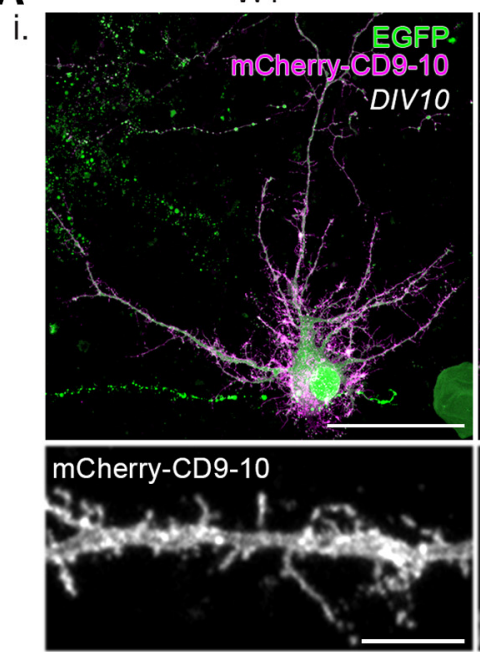

ii.

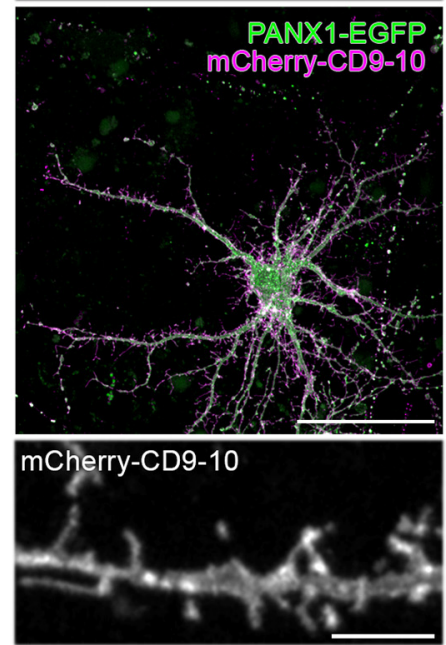

Panx1 KO

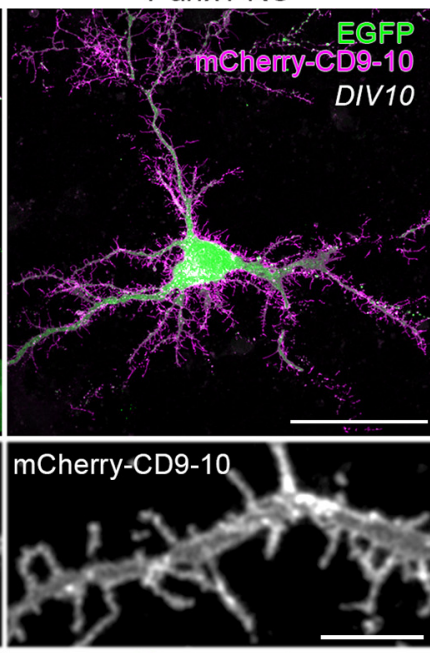

PANX1-EGFP

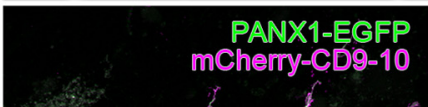

B

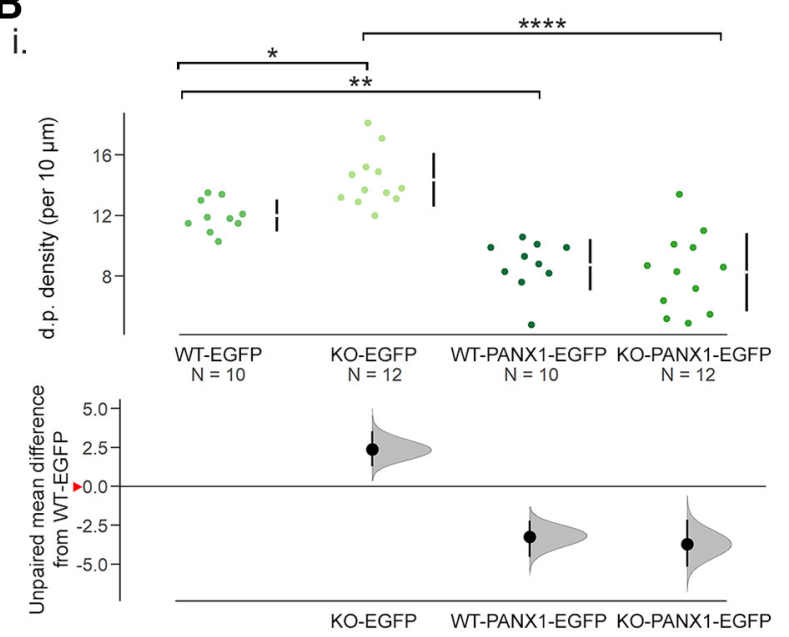

ii.

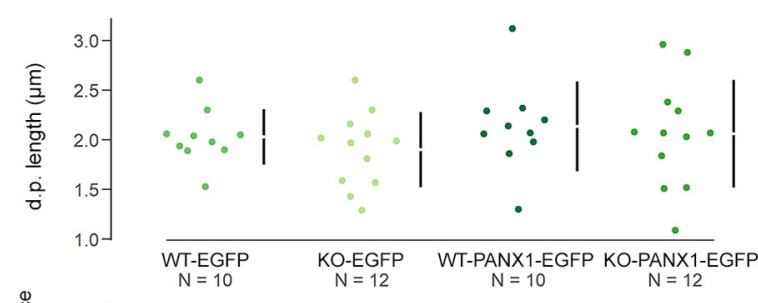

Figure 2. PANX1-EGFP expression rescued the increase in dendritic protrusion density associated with Panx1 KO. A, Representative maximum intensity projections of WT and Panx1 KO cultured cortical neurons transfected with mCherry-CD9-10 and either EGFP (Ai) or PANX1-EGFP (Aii) as well as cropped images of their respective dendritic segments from a primary neurite. Scale bars: 50 and $5 \mu \mathrm{m}$. B. Effect of PANX1 expression on dendritic protrusion density and length in developing cortical neurons transfected with mCherry-CD9-10 and either EGFP or PANX1-EGFP using Cumming estimation plots. Bi, With EGFP expression, dendritic protrusion density was higher with Panx1 KO neurons (WT-EGFP: $12.0 \pm 0.3$ dendritic protrusions per $10 \mu \mathrm{m}$; Panx1 KOEGFP: $14.4 \pm 0.5$ dendritic protrusions per $10 \mu \mathrm{m}, p=0.03517$, two-way ANOVA with Bonferroni's multiple comparison test $\left.{ }^{\mathrm{b} 1}\right)$. With PANX1-EGFP expression, dendritic protrusion density was decreased in both WT and Panx1 KO neurons (WT-PANX1-EGFP: $8.8 \pm 0.5$ dendritic protrusions per $10 \mu \mathrm{m}, \rho=0.00268$; Panx1 KO PANX1-EGFP: $8.3 \pm 0.8$ dendritic protrusions per $10 \mu \mathrm{m}$, $p<0.0001$, two-way ANOVA with Bonferroni's multiple comparison test $\left.{ }^{\mathrm{b} 1}\right)$. Bii, No significant differences in dendritic protrusion length were found between groups (WT-EGFP: $2.0 \pm 0.3 \mu \mathrm{m}$; Panx1 KO-EGFP: $1.9 \pm 0.4 \mu \mathrm{m}, p>0.9999$, two-way ANOVA with Bonferroni's multiple comparison test ${ }^{\mathrm{b} 2}$; WT-PANX1-EGFP: $2.1 \pm 0.1 \mu \mathrm{m}$; Panx1 KO PANX1-EGFP: $2.1 \pm 0.2 \mu \mathrm{m}, p>0.9999$, twoway ANOVA with Bonferroni's multiple comparison test). Data are presented as mean $\pm S D . N=$ cells, all analyzed cells were obtained from three independent cultures. Effect sizes are reported in the main text and Table 2. Red arrowheads on the $y$-axis on the bottom panel of Cumming estimation plots represent WT-EGFP means. d.p., dendritic protrusion; $* * * *<0.0001, * *<0.01, *<0.05$. This figure was modified from the PhD Thesis of J.C.S.-A, University of Victoria (Sanchez-Arias, 2020), found at http://hdl.handle. net/1828/11714.

expressing neurons within Panx1 KO cultures only (KOEGFP vs KO-PANX1-EGFP: $-10.4 \% \quad[95 \mathrm{Cl}-14 \%$; $-6.58 \%], p=0.00028^{d 1}$; Fig. $\left.5 A, B i\right)$. We next measured the overall gain and loss of dendritic protrusions (turnover) by adding together dendritic protrusion formation, elimination, and lability, divided by the total number of dendritic protrusions that were present at the beginning of the analysis (time 0). Turnover was significantly reduced in Panx1 KO compared with WT neurons (effect size WT-EGFP vs KO-EGFP: $-4.48 \%[95 \mathrm{Cl}-7.73 \% ;-2.01 \%], p=0.0092^{\mathrm{d} 2}$; Fig. 5Bii) in EGFP-expressing control cultures. PANX1-EGFP expression significantly increased turnover in Panx1 KO cultures (effect size KO-EGFP vs KO-PANX1-EGFP: $5.24 \%$ [95Cl 2.87\%; $8.66 \%], p=0.0027^{\mathrm{d} 2}$ ). Finally, we added together the four fundamental metrics (formation, elimination, lability, and motility) to obtain an overall metric for the change in dendritic 
A

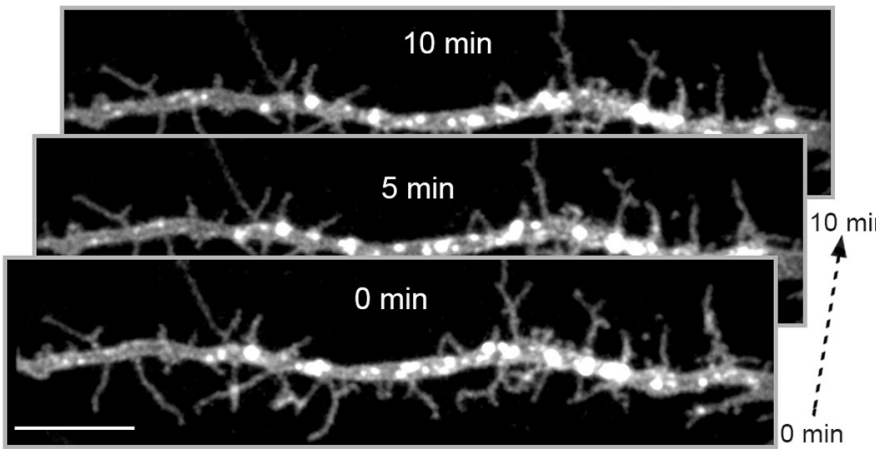

Threshold application (i.e., make binary) Creation of outlines from threshold images

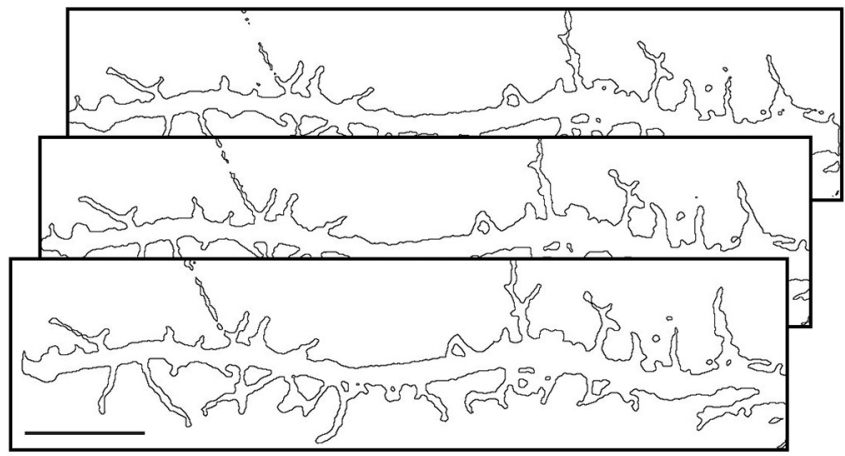

B

Temporal colour-code 0 min $\square 10 \mathrm{~min}$

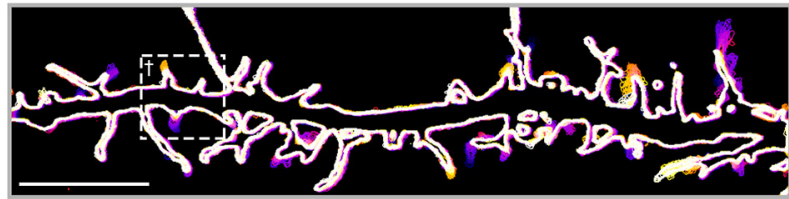

C
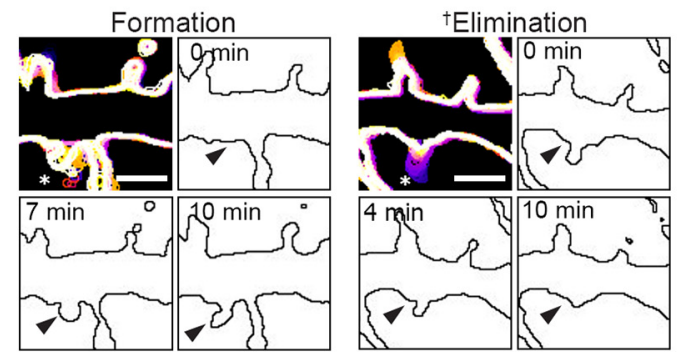

Lability

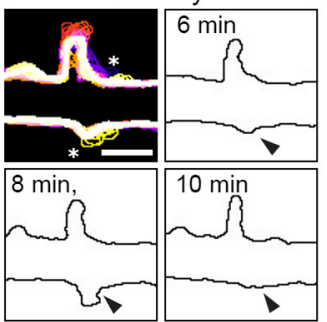

Motility

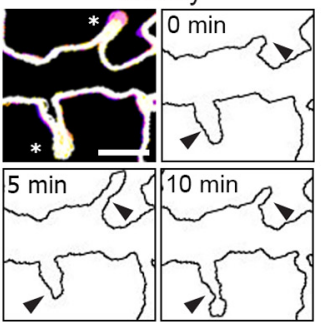

Figure 3. Novel methods for measurement of dendritic protrusion dynamics in living neurons. Ten-minute time lapses were acquired by imaging dendrite segments from cortical neurons every $5 \mathrm{~s}$. Note that this a DIV10 WT cortical neuron transfected with mCherry-CD9-10 and PANX1-EGFP; only mCherry-CD9-10 is shown. The dimensionality of these recordings was reduced by creating maximum $z$-projections. Images were thresholded to create outlines $(\boldsymbol{A}$; scale bar: $10 \mu \mathrm{m})$, which were temporally color coded $(\boldsymbol{B}$; scale bar: $10 \mu \mathrm{m})$, allowing the visualization of various events, such as the percentage of dendritic protrusion (relative to time 0 ) undergoing formation (de novo appearance), elimination (complete disappearance by the end of the time lapse), lability (appearance and disappearance by the end of the time lapse), and motility (incomplete shrinkage or growth to an existing protrusion) shown in $\boldsymbol{C}$ (scale bar: $2 \mu \mathrm{m}$ ). Note that examples in $\boldsymbol{C}$ (cropped to highlight the event in question with $(*)$ denoting the protrusion events) come from different cultures and different genotypes all at DIV10 transfected with mCherry-CD9-10 and either EGFP or Panx1-EGFP at DIV6. Note that the example provided here for dendritic protrusion elimination $(\boldsymbol{t})$ in the box in part $\boldsymbol{C}$ comes from the larger neurite depicted in panel $\boldsymbol{B}$. Also note that the data shown in Figure 4 includes quantification from the examples depicted in this figure. For further details, see Materials and Methods. This figure was modified from the PhD thesis of J.C.S.-A, University of Victoria (Sanchez-Arias, 2020), found at http://hdl.handle.net/1828/11714.

protrusion movement ( $\Delta$ movement). Within EGFP control expressing cultures, $\Delta$ movement was significantly reduced in Panx1 KO neurons compared with WT controls (effect size WT-EGFP vs KO-EGFP: -17.8 [95Cl -23.6 ; -11.9$]$, $p<0.0001$; Fig. 5Biii). Transient PANX1-EGFP expression resulted in increased $\Delta$ movement in Panx1 KO cultures only (effect size KO-EGFP vs KO-PANX1-EGFP: 22.8 [95Cl 15; 30.4], $p=0.00033^{\mathrm{d} 3}$ ). Altogether, these results suggest that Panx1 KO neuron dendritic protrusions are more stable and that this effect is rescued by transient expression of PANX1EGFP.

\section{Discussion}

\section{PANX1 increases dendritic protrusion movement}

Previous work showed that Panx1 KO was associated with an increase in dendritic spine density in situ and in vitro (Sanchez-Arias et al., 2019). However, it was unclear whether the increased dendritic spine density arose from additional formation and/or enhanced stability of spines. In order to investigate how PANX1 regulates the stability of dendritic protrusions, the precursors to dendritic spines, we developed a novel approach consisting of sparse expression of a membrane bound fluorescent protein, mCherry-CD9-10. This method significantly improved detection of dendritic protrusions over commonly used cytoplasmic fluorescent protein expression approaches (Mancuso et al., 2013). This novel methodology enabled us to discover a reciprocal relationship between PANX1 expression levels and dendritic protrusion density and stability. Taken together with the previous findings, this work suggests that PANX1 regulation of dendritic protrusion dynamics contributed to the increase in cortical dendritic spine density previously observed with Panx1 KO. Moreover, these findings suggest that developmental downregulation of PANX1 likely contributes to increased spine stability underlying spine formation in vivo during the first four postnatal weeks of brain development.

Transient expression of PANX1-EGFP significantly affected dendritic protrusion dynamics in Panx1 KO 
A

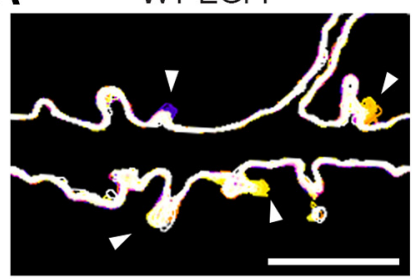

Panx1 KO-EGFP

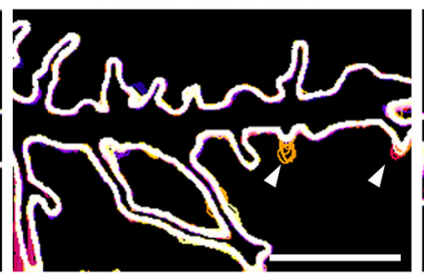

B

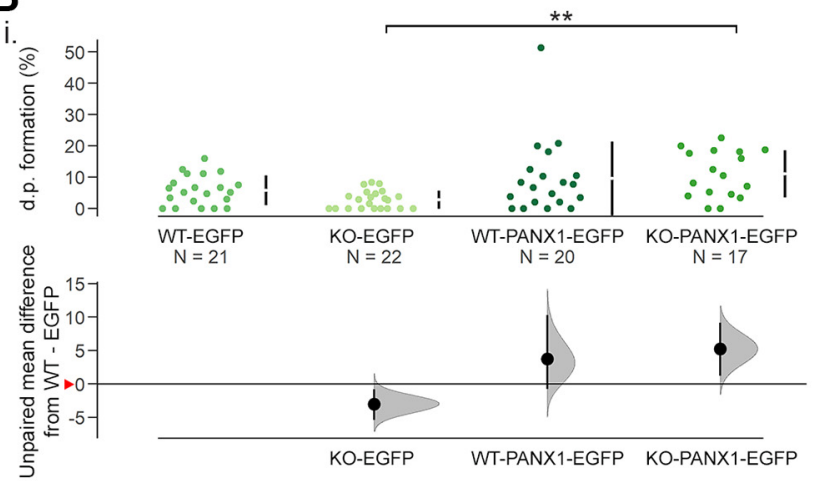

iii.

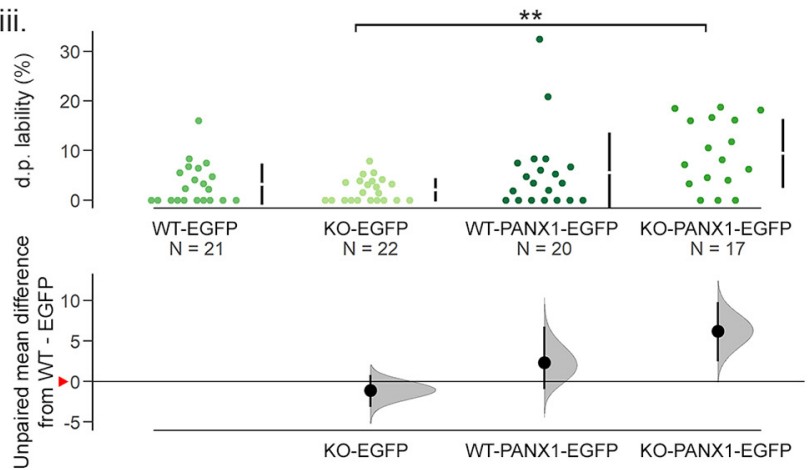

WT-PANX1-EGFP
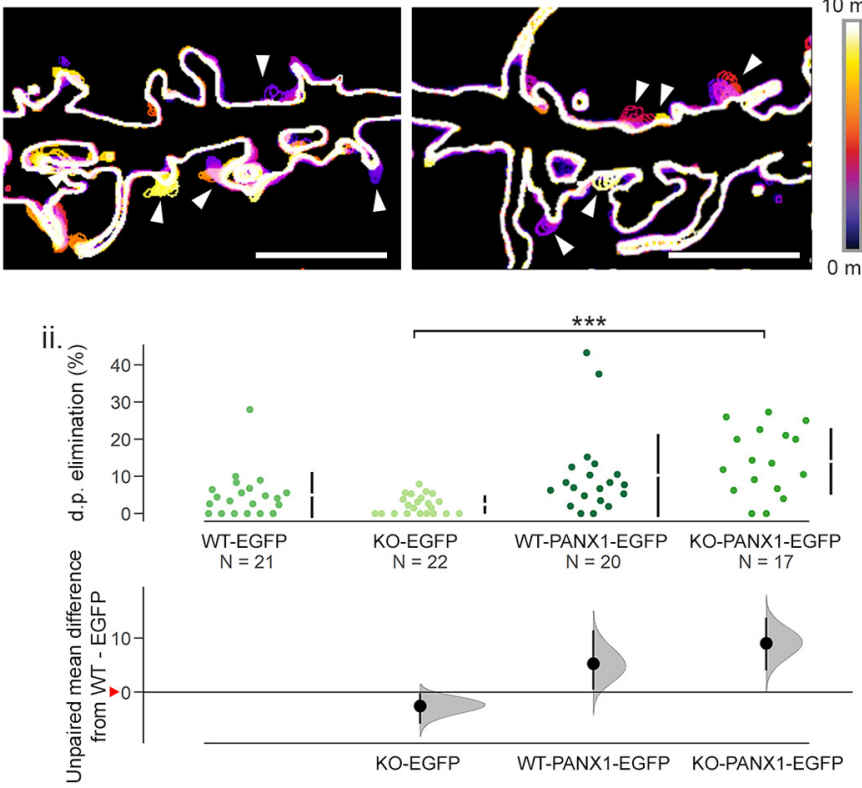

iv.

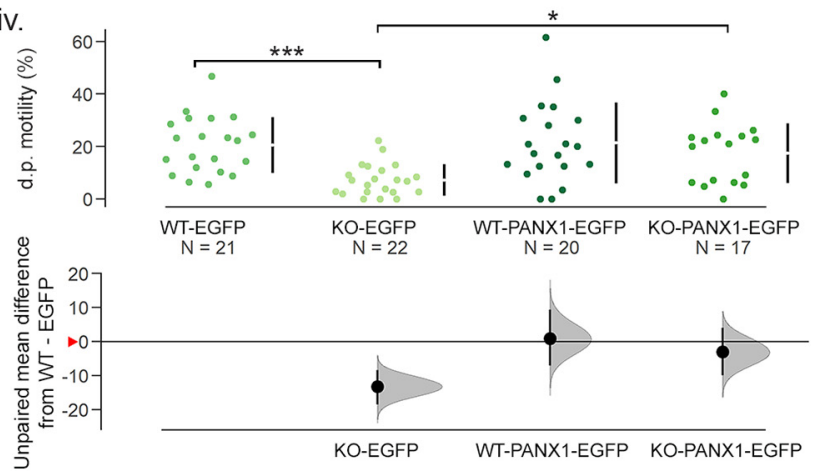

Figure 4. Metrics of dendritic protrusion dynamics correlated with PANX1 expression levels. $\boldsymbol{A}$, Representative color-coded outlines of WT and Panx1 KO neurons transfected with mCherry-CD9-10 and either EGFP or Panx1-EGFP showing examples of dendritic protrusion formation, elimination, lability, and motility events (arrowheads). These examples are cropped from the full regions of analysis from primary neurites. B, Effect of PANX1 expression on dendritic protrusion formation, elimination, lability, and motility in WT and Panx $1 \mathrm{KO}$ using Cumming estimation plots. Bi, Dendritic protrusion formation was significantly higher in Panx $1 \mathrm{KO}$ neurons transiently expressing PANX1-EGFP compared with those expressing EGFP (KO-EGFP: $0.2 \pm 0.1 \%$, KO-PANX1-EGFP: $4.6 \pm$ $1.3 \%, p=0.0028$, Kruskal-Wallis test ${ }^{c 1}$ ). No significant differences were observed between genotypes in EGFP-expressing neurons (WT-EGFP: $1.7 \pm 0.7 \%$; Panx1 KO-EGFP: $0.2 \pm 0.1 \%, p=0.2267$, Kruskal-Wallis test ${ }^{\mathrm{c} 1}$ ). Bii, Similarly, only transient expression of PANX1-EGFP in Panx1 KO neurons increased dendritic protrusion elimination (KO-EGFP: $0.3 \pm 0.15 \%$; KO-PANX1-EGFP: $4.6 \pm$ $1.28 \%, p=0.00024$, Kruskal-Wallis test ${ }^{\mathrm{C} 2}$ ). No significant differences were found between EGFP and PANX1-EGFP expressing WT cells $\left(p=0.62307^{\mathrm{C2}}\right)$. Biii, Dendritic protrusion lability was higher in Panx1 KO neurons transfected with PANX1-EGFP (KO-EGFP: $2.1 \pm 0.5 \%$; KO-PANX1-EGFP: $9.4 \pm 1.7 \%, p=0.0034$, Kruskal-Wallis test ${ }^{\mathrm{C} 3}$ ), beyond that observed in WT expressing EGFP control $\left(p=0.0291\right.$, Kruskal-Wallis test $\left.{ }^{\mathrm{c} 3}\right)$. Transient expression of PANX1-EGFP in WT neurons had no significant effects $(p>$ $0.9999^{\mathrm{c} 3}$ ). Biv, Dendritic protrusion motility was significantly reduced in Panx1 KO neuron expressing EGFP control (WT-EGFP: 20.5 $\pm 2.3 \%$; KO-EGFP: $7.2 \pm 1.3 \%, p=0.00016$, Kruskal-Wallis test ${ }^{\mathrm{c} 4}$ ). Transient PANX1-EGFP expression increased dendritic protru-

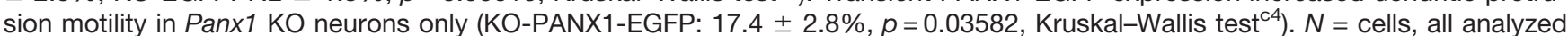
cells were obtained from three independent cultures. Effect sizes are reported in the main text and Table 2. Red arrowheads on the $y$-axis on the bottom panel of Cumming estimation plots represent WT-EGFP means. d.p., dendritic protrusion; $* * *<0.001$, $* *<0.01, *<0.05$. This figure was modified from the PhD thesis of J.C.S.-A, University of Victoria (Sanchez-Arias, 2020), found at http://hdl.handle.net/1828/11714.

cultures but had less of an impact in WT cultures. The dampened impact of PANX1-EGFP in WT neurons implied the presence of a ceiling effect. Saturation of PANX1mediated increases in dendritic protrusion movement may have resulted from limited availability of additional machinery for proper PANX1 trafficking and/or auto- regulation via ATP-dependent internalization (i.e., increase in PANX1 leading to increase in ATP release feeding back to internalize PANX1; Boyce et al., 2015; Boyce and Swayne, 2017). Alternatively, effects of supplementary PANX1 were perhaps constrained by limited availability of interacting partners or saturation of downstream 

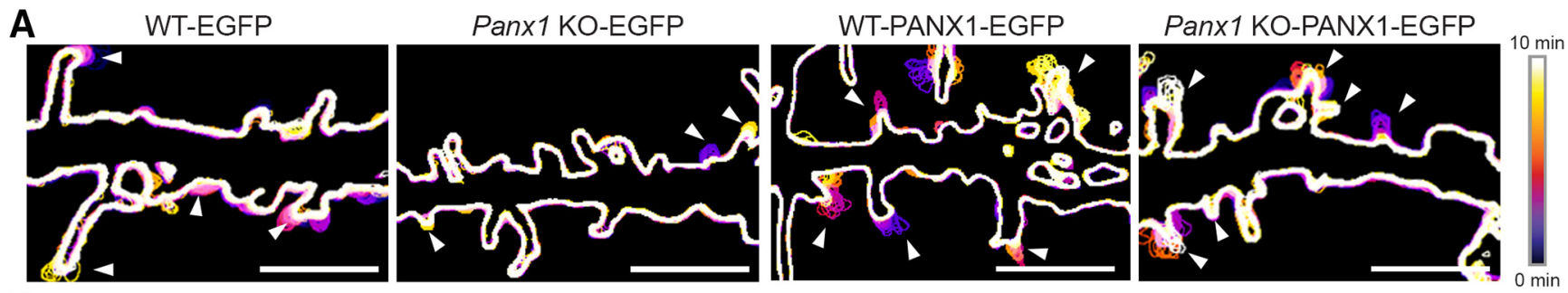

B
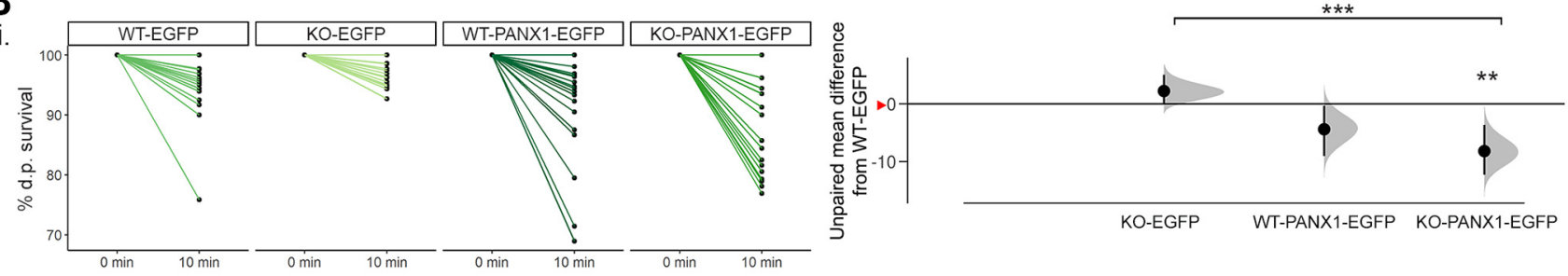

ii.

$$
\text { Turnover }(\%)=\frac{\text { Elimination }+ \text { Formation }+ \text { Lability }}{\text { dendritic protrusions (d.p.) at time } 0 \mathrm{~min}} \times 100 \%
$$$$
\text { ** }
$$

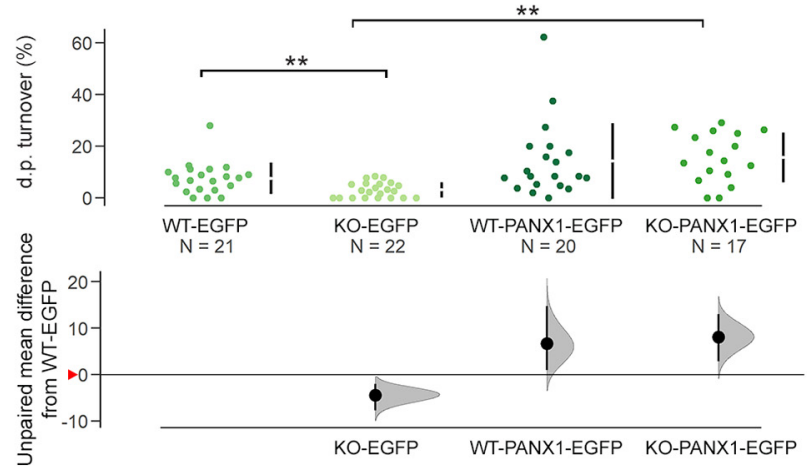

iii.

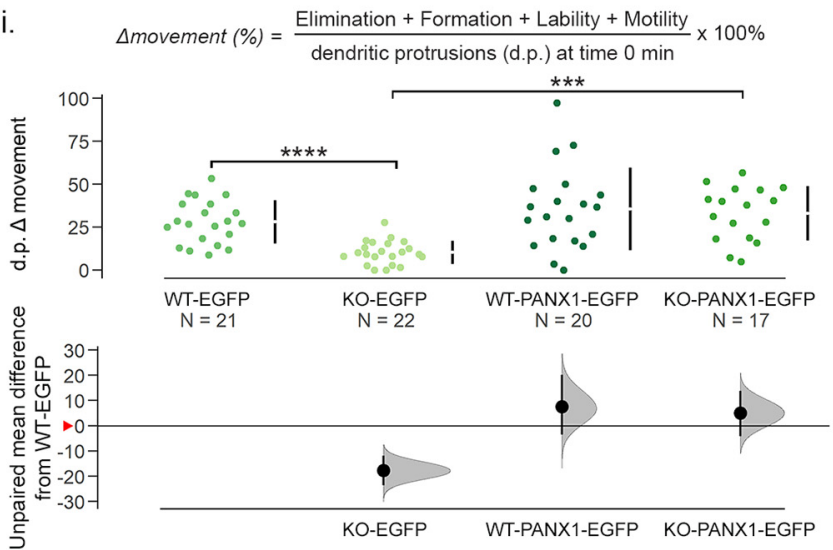

Figure 5. PANX1 increased dendritic protrusion turnover and overall movement. $\boldsymbol{A}$, Representative color-coded outlines of WT and Panx1 KO neurons transfected with mCherry-CD9-10 and either EGFP or PANX1-EGFP showing examples of dendritic protrusion movement (arrowheads). These examples are cropped from the full regions of analysis from primary neurites. $\boldsymbol{B}$, Cumming estimation plots of dendritic protrusion second order metrics: survival fraction, turnover, and overall change in movement ( $\Delta$ movement). Bi, Transient PANX1 expression in WT and Panx1 KO neurons decreased the survival fraction of dendritic protrusions; however, this was only statistically significant in Panx1 KO neurons (WT-EGFP: $94.5 \pm 1.2 \%$; WT-PANX1-EGFP: $91.1 \pm 1.9 \%, p=0.2034^{\mathrm{d} 1}$; PANX1-EGFP: $97.7 \pm$ 0.5\%; Panx1 KO-PANX1-EGFP: $87.3 \pm 1.9 \%, p=0.00028$, Kruskal-Wallis test ${ }^{\mathrm{d} 1}$ ). Bii, In the EGFP-control-expressing group, dendritic protrusion turnover was reduced in Panx1 KO neurons (WT-EGFP: $7.5 \% \pm 1.3$; PANX1-EGFP: $3.1 \pm 0.6 \%, p=0.0092$, Kruskal-Wallis test $^{\mathrm{d} 2}$ ). Transient expression of PANX1 significantly increased dendritic protrusion turnover in Panx1 KO neurons but not in WT neurons (WT-PANX1-EGFP: $14.2 \pm 3.3 \%, p>0.9999$; Panx1 KO-PANX1-EGFP: $15.6 \pm 2.34 \%, p=0.0027$, Kruskal-Wallis test ${ }^{\mathrm{d} 2}$ ). Biii, Dendritic protrusion overall movement change ( $\Delta$ movement) was reduced in Panx1 KO neurons (WT-EGFP: $28 \pm 2.8 \%$; KO-EGFP: 10.3 $\pm 1.5 \%$, $p<0.0001$, Kruskal-Wallis test ${ }^{\mathrm{d} 3}$ ). PANX1-EGFP expression increased $\Delta$ movement in both WT (WT-PANX1-EGFP: $35.5 \pm 5.4 \%$ ) and Panx1 KO neurons; however, this effect was only significant in Panx1 KO neurons (KO-PANX1-EGFP: $33 \pm 3.8 \%, p=0.00033$, KruskalWallis test ${ }^{\mathrm{d} 3}$ ). $N=$ cells, all analyzed cells were obtained from three independent cultures. Effect sizes are reported in the main text and Table 2. Red arrowheads on the $y$-axis on the bottom panel of Cumming estimation plots represent WT-EGFP means. d.p., dendritic protrusion; $* * * *<0.0001, * * *<0.001, * *<0.01$. This figure was modified from the PhD thesis of J.C.S.-A, University of Victoria (SanchezArias, 2020), found at http://hdl.handle.net/1828/11714.

purinergic signaling pathways (Abbracchio et al., 2009; Bhalla-Gehi et al., 2010; Wicki-Stordeur and Swayne, 2013; Dahl, 2015; Yang et al., 2015; Xu et al., 2018).

PANX1 regulation of dendritic protrusion stability could underlie neuronal network and synaptic plasticity changes associated with PANX1 disruption. For example, Rafael et al. (2020) recently reported that Panx1 KO hippocampal neurons treated with tetrodotoxin (TTX) for $36 \mathrm{~h}$ (in a model of "chronic inactivity") did not scale up their synapses accordingly, hinting that loss of PANX1 altered homeostatic plasticity mechanisms. Interestingly, the lack of synaptic scaling was seen both in Panx1 KO neuron-glial co-cultures as well as Panx1 KO neurons co-cultured with WT glia. On the other hand, WT neuron-glial co-cultures and WT neurons co-cultured with Panx1 KO glia scaled up their synapses in the presence of chronic inactivity induced by TTX treatment. These findings suggest that neuronal, but not glial, PANX1 plays a role in the regulation of synaptic homeostatic plasticity and raises the possibility that increased stability of dendritic protrusions in Panx1 $\mathrm{KO}$ could be a contributing factor to these altered synaptic plasticity adaptations (Holtmaat and Svoboda, 2009; O’Donnell et al., 2011; Yin and Yuan, 2015). Future studies should aim to elucidate the molecular mechanism 
Table 2: Statistical table

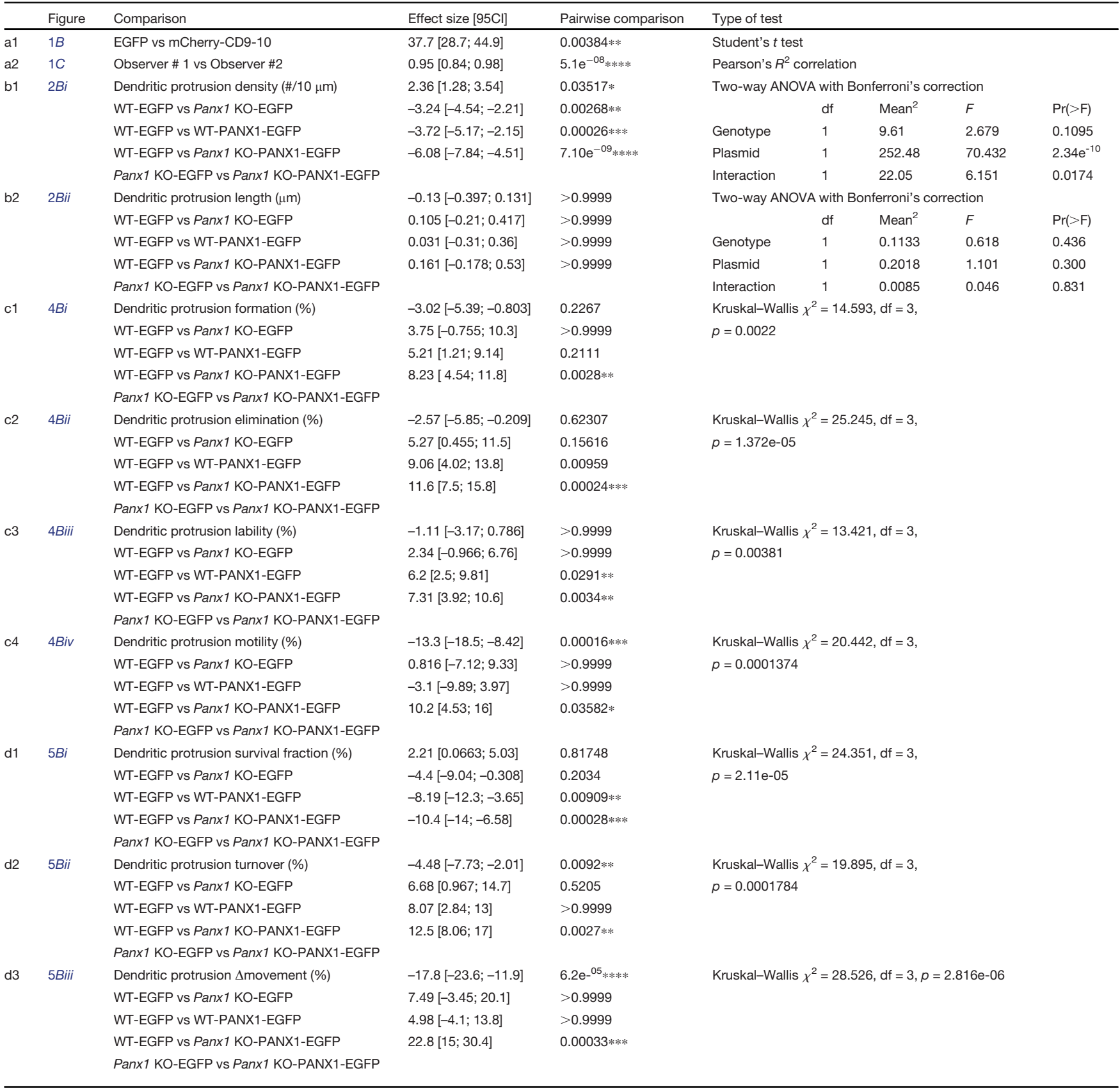

Significance codes: $* * * *<0.0001, * * *<0.001, * *<0.01, *<0.05$.

underlying PANX1 regulatory role(s) in the maturation and maintenance of excitatory synapses throughout development and in the context of neuron-neuron (excitatory-inhibitory balance) and neuron-glial interactions (astrocytes, oligodendrocytes, microglia).

\section{PANX1 regulation of dendritic spines is consistent with its role in synaptic plasticity and neurodevelopment}

Our findings provide important new insight into recent studies demonstrating that PANX1 regulates brain and cognitive development. Panx1 KO has been associated with altered hippocampal plasticity (LTP facilitation and LTD deficits), impaired memory flexibility, and larger cortical neuron network ensembles and spine densities (Prochnow et al., 2012; Ardiles et al., 2014; Gajardo et al., 2018; Sanchez-Arias et al., 2019). Dendritic spine abnormalities have been observed in several neurodevelopmental disorders (Forrest et al., 2018; Lima-Caldeira et al., 2019). Brain enriched, autism spectrum disorders (ASDs)associated single nucleotide polymorphisms (SNPs) have been associated with changes in PANX1 expression levels (Davis et al., 2012). A germline PANX1 SNP was associated with intellectual disability (Shao et al., 2016). PANX1 is also linked to neurodevelopmental disorders 
indirectly through its interacting partner CRMP2 and its downstream signaling effectors and interactors, purinergic receptors (Boyce et al., 2015; Boyce and Swayne, 2017; for review, see Swayne and Boyce, 2017). CRMP2 auto-antibodies have been implicated in ASD (Braunschweig et al., 2013), and brain-specific deletion of the gene encoding for CRMP2 resulted in decreased dendritic spine density and reduced LTP (Zhang et al., 2016), while suramin treatment corrected synaptic and behavioral phenotypes in the Fmr1 KO mouse (Naviaux et al., 2013, 2015, 2017).

\section{Limitations}

Immature neurons contain mainly filopodia-like structures and immature spines that lack fully developed postsynaptic densities (PSDs) (Berry and Nedivi, 2017). In order to facilitate observation of a substantial number of dendritic protrusions simultaneously, we selected a single immature time point (DIV10). We used this strategy because dendritic protrusions are the precursors to mature spines, although it should be noted that not all protrusions develop into spines. With this in mind, an important extension of the current study will be to assess synaptic markers within the same cells in a longitudinal approach (i.e., over multiple days), as this would enable tracking of dendritic protrusions that eventually develop into spines at a more mature DIV, as well as, retrospective grouping of our dendritic protrusion dynamics data based on the "fate" of the dendritic protrusions (i.e., attributes of those that become spines vs those that do not). Somewhat surprisingly, Panx1 KO cortical neurons already exhibited increased dendritic protrusion density at this relatively immature analysis time point, suggesting that PANX1 begins to regulate dendritic protrusions, the precursors to dendritic spines, early in neuronal development. Previous work revealed a proportional increase in PSD-95positive spines associated with $P a n \times 1 \mathrm{KO}$ at a more mature DIV (DIV12), suggesting the increase in dendritic protrusion density associated with Panx $1 \mathrm{KO}$ indeed leads to a corresponding increase in dendritic spine density. In addition to facilitating tracking the fate of the dendritic protrusions, assessment of PSD-95 trafficking into spines, as a readout of maturation, would also permit simultaneous investigation of the mechanistic underpinnings of PANX1 regulation of dendritic spine formation. Spine formation relies on microtubule dynamics (Hu et al., 2011), and CRMP2, a PANX1 interacting protein, is a well-known regulator of microtubule dynamics as well as dendritic spine formation (Jin et al., 2016; Zhang et al., 2016). Additionally, it will be important to examine functionally similar proteins or mechanisms that could compensate for the loss of PANX1, such as changes in expression levels of other pannexins, functionally related connexins or purinergic receptors. Future pharmacological and genetic experiments disrupting PANX1 and its interactome will continue to enhance our understanding of PANX1 regulation of neuronal development.

There are some additional limitations associated with our KO model that are important to note. The original Pan $\times 1^{f / f}$ strain was developed on a $129 \times 1 / \mathrm{SvJ}$ background, and Panx1 KO was generated via a cross with a CMV-Cre line (Dvoriantchikova et al., 2012), which were then backcrossed over 10 generations onto the C57BI/6J background. The original strain contained a loss of function mutation in Casp4 (Casp11) and was predicted to have variants in Mmp1a, Olfr832, Fbxl12, ENSMUSG00000095186, and ENSMUSG00000095891 (Vanden Berghe et al., 2015). We cannot rule out the possibility that these passenger mutations could have been present in the global Panx $1 \mathrm{KO}$ and not their control littermates; however, we also compared a conditional Panx 1 KO $\left(\operatorname{Pan} x 1^{f / f} ; E m \times 1^{1 R E S-C r e}\right)$ strain with control Panx $1^{f / f}$ littermates (i.e., same genetic background in conditional $\mathrm{KO}$ and control littermates), which yielded the same result in terms of dendritic spine densities, suggesting that this phenotype can be attributed to deletion of Panx1. Furthermore, transient expression of PANX1-EGFP rescued the change in dendritic protrusion density associated with Panx1 KO, further supporting our findings from Panx1 KO mouse that PANX1 inversely correlates with regulation of dendritic spine formation.

\section{Conclusions}

In summary, this work has significantly advanced our understanding of the role of PANX1 in dendritic spine development, identifying it as a regulator of dendritic protrusion dynamics. PANX1 promotes dendritic protrusion movement and is associated with greater dendritic protrusion instability. The role of PANX1 in the regulation of this process that relies inherently on complex cytoskeletal dynamics is consistent with its previously identified interactions with ARP2/3 and CRMP2, known to control both cytoskeletal dynamics and dendritic spine development. Moreover, these novel findings presented here also align with the marked downregulation of cortical PANX1 expression levels in mice that coincides with spine formation during the first four postnatal weeks. Together, these findings reveal an important role for PANX1 in regulating dendritic protrusion dynamics in developing cortical neurons.

\section{References}

Abbracchio MP, Burnstock G, Verkhratsky A, Zimmermann H (2009) Purinergic signalling in the nervous system: an overview. Trends Neurosci 32:19-29.

Araya R, Vogels TP, Yuste R (2014) Activity-dependent dendritic spine neck changes are correlated with synaptic strength. Proc Natl Acad Sci USA 111:E2895-E2904.

Ardiles AO, Flores-Muñoz C, Toro-Ayala G, Cárdenas AM, Palacios AG, Muñoz P, Fuenzalida M, Sáez JC, Martínez AD (2014) Pannexin 1 regulates bidirectional hippocampal synaptic plasticity in adult mice. Front Cell Neurosci 8:326.

Bardy $\mathrm{C}, \mathrm{M}$ van den $\mathrm{H}$, Eames $\mathrm{T}$, Marchand $\mathrm{C}$, Hernandez RV, Kellogg M, Gorris M, Galet B, Palomares V, Brown J, Bang AG, Mertens J, Böhnke L, Boyer L, Simon S, Gage FH (2015) Neuronal medium that supports basic synaptic functions and activity of human neurons in vitro. Proc Natl Acad Sci USA 112:E2725E2734.

Bernard C (2019) Changing the way we report, interpret, and discuss our results to rebuild trust in our research. eNeuro 6: ENEURO.0259-19.2019.

Berry KP, Nedivi E (2017) Spine dynamics: are they all the same? Neuron 96:43-55.

Bhalla-Gehi R, Penuela S, Churko JM, Shao Q, Laird DW (2010) Pannexin1 and Pannexin3 delivery, cell surface dynamics, and cytoskeletal interactions. J Biol Chem 285:9147-9160. 
Boyce AKJ, Swayne LA (2017) P2X7 receptor cross-talk regulates ATP-induced pannexin 1 internalization. Biochem J 474:21332144.

Boyce AKJ, Kim MS, Wicki-Stordeur LE, Swayne LA (2015) ATP stimulates pannexin 1 internalization to endosomal compartments. Biochem J 470:319-330.

Boyce AKJ, Epp AL, Nagarajan A, Swayne LA (2018) Transcriptional and post-translational regulation of pannexins. Biochim Biophys Acta Biomembr 1860:72-82.

Braunschweig D, Krakowiak P, Duncanson P, Boyce R, Hansen RL, Ashwood P, Hertz-Picciotto I, Pessah IN, Van de Water J (2013) Autism-specific maternal autoantibodies recognize critical proteins in developing brain. Transl Psychiatry 3:e277.

Calin-Jageman RJ, Cumming G (2019) Estimation for Better Inference in Neuroscience. eNeuro 6:ENEURO.0205-19.2019.

Chiu YH, Schappe MS, Desai BN, Bayliss DA (2018) Revisiting multimodal activation and channel properties of pannexin 1. J Gen Physiol 150:19-39.

Dahl G (2015) ATP release through pannexon channels. Phil Trans R Soc B 370:20140191.

Davis LK, Gamazon ER, Kistner-Griffin E, Badner JA, Liu C, Cook EH, Sutcliffe JS, Cox NJ (2012) Loci nominally associated with autism from genome-wide analysis show enrichment of brain expression quantitative trait loci but not lymphoblastoid cell line expression quantitative trait loci. Mol Autism 3:3.

Dunaevsky A, Tashiro A, Majewska A, Mason C, Yuste R (1999) Developmental regulation of spine motility in the mammalian central nervous system. Proc Natl Acad Sci USA 96:13438-13443.

Dvoriantchikova G, Ivanov D, Barakat D, Grinberg A, Wen R, Slepak VZ, Shestopalov VI (2012) Genetic ablation of Pannexin1 protects retinal neurons from ischemic injury. PLoS One 7:e31991.

Fiala JC, Feinberg M, Popov V, Harris KM (1998) Synaptogenesis via dendritic filopodia in developing hippocampal area CA1. J Neurosci 18:8900-8911.

Forrest MP, Parnell E, Penzes P (2018) Dendritic structural plasticity and neuropsychiatric disease. Nat Rev Neurosci 19:215-234.

Gajardo I, Salazar CS, Lopez-Espíndola D, Estay C, Flores-Muñoz C, Elgueta C, Gonzalez-Jamett AM, Martínez AD, Muñoz P, Ardiles ÁO (2018) Lack of pannexin 1 alters synaptic GluN2 subunit composition and spatial reversal learning in mice. Front Mol Neurosci 11:114.

Grolemund G, Wickham H (2017) R for data science: visualize, model, transform, and import data. Available from https://r4ds. had.co.nz/.

Ho J, Tumkaya T, Aryal S, Choi H, Claridge-Chang A (2019) Moving beyond $P$ values: data analysis with estimation graphics. Nat Methods 16:565-566.

Holtmaat A, Svoboda K (2009) Experience-dependent structural synaptic plasticity in the mammalian brain. Nat Rev Neurosci 10:647658.

Holtmaat AJGD, Trachtenberg JT, Wilbrecht L, Shepherd GM, Zhang X, Knott GW, Svoboda K (2005) Transient and persistent dendritic spines in the neocortex in vivo. Neuron 45:279-291.

$\mathrm{Hu}$ X, Ballo L, Pietila L, Viesselmann C, Ballweg J, Lumbard D, Stevenson M, Merriam E, Dent EW (2011) BDNF-induced increase of PSD-95 in dendritic spines requires dynamic microtubule Invasions. J Neurosci 31:15597-15603.

Jin X, Sasamoto K, Nagai J, Yamazaki Y, Saito K, Goshima Y, Inoue T, Ohshima T (2016) Phosphorylation of CRMP2 by Cdk5 regulates dendritic spine development of cortical neuron in the mouse hippocampus. Neural Plast 2016:6790743.

Lima-Caldeira G, Peça J, Carvalho AL (2019) New insights on synaptic dysfunction in neuropsychiatric disorders. Curr Opin Neurobiol $57: 62-70$.

Mancuso JJ, Chen Y, Li X, Xue Z, Wong STC (2013) Methods of dendritic spine detection: from Golgi to high-resolution optical imaging. Neuroscience 251:129-140.

McDonald J (2014) Handbook of biological statistics. Baltimore: Sparky House.
Naviaux RK, Zolkipli Z, Wang L, Nakayama T, Naviaux JC, Le TP, Schuchbauer MA, Rogac M, Tang Q, Dugan LL, Powell SB (2013) Antipurinergic therapy corrects the autism-like features in the poly (IC) mouse model. PLoS One 8:e57380.

Naviaux JC, Wang L, Li K, Bright AT, Alaynick WA, Williams KR, Powell SB, Naviaux RK (2015) Antipurinergic therapy corrects the autism-like features in the fragile $X$ (Fmr1 knockout) mouse model. Mol Autism 6:1.

Naviaux RK, Curtis B, Li K, Naviaux JC, Bright AT, Reiner GE, Westerfield M, Goh S, Alaynick WA, Wang L, Capparelli EV, Adams C, Sun J, Jain S, He F, Arellano DA, Mash LE, Chukoskie L, Lincoln A, Townsend J (2017) Low-dose suramin in autism spectrum disorder: a small, phase I/II, randomized clinical trial. Ann Clin Transl Neurol 4:491-505.

O'Donnell C, Nolan MF, van Rossum MCW (2011) Dendritic spine dynamics regulate the long-term stability of synaptic plasticity. $J$ Neurosci 31:16142-16156.

Penuela S, Bhalla R, Gong X-Q, Cowan KN, Celetti SJ, Cowan BJ, Bai D, Shao Q, Laird DW (2007) Pannexin 1 and pannexin 3 are glycoproteins that exhibit many distinct characteristics from the connexin family of gap junction proteins. J Cell Sci 120:3772-3783.

Polanco JC, Li C, Durisic N, Sullivan R, Götz J (2018) Exosomes taken up by neurons hijack the endosomal pathway to spread to interconnected neurons. Acta Neuropathol Commun 6:10.

Prange O, Murphy TH (2001) Modular transport of postsynaptic density-95 clusters and association with stable spine precursors during early development of cortical neurons. J Neurosci 21:93259333.

Prochnow N, Abdulazim A, Kurtenbach S, Wildförster V, Dvoriantchikova G, Hanske J, Petrasch-Parwez E, Shestopalov VI, Dermietzel R, Manahan-Vaughan D, Zoidl G (2012) Pannexin1 stabilizes synaptic plasticity and is needed for learning. PLoS One 7: e51767.

Rafael A, Cairus A, Tizzoni M, Abudara V, Vitureira N (2020) Glial ATP and large pore channels modulate synaptic strength in response to chronic inactivity. Mol Neurobiol 57:2856-2869.

Ray A, Zoidl G, Weickert S, Wahle P, Dermietzel R (2005) Site-specific and developmental expression of pannexin1 in the mouse nervous system. Eur J Neurosci 21:3277-3290.

Sala C, Segal M (2014) Dendritic spines: the locus of structural and functional plasticity. Physiol Rev 94:141-188.

Sanchez-Arias JC (2020) Pannexin 1 regulates dendritic spines in developing cortical neurons. University of Victoria. Available from https://dspace.library.uvic.ca//handle/1828/11714.

Sanchez-Arias JC, Liu M, Choi CSW, Ebert SN, Brown CE, Swayne LA (2019) Pannexin 1 regulates network ensembles and dendritic spine development in cortical neurons. eNeuro 6:ENEURO.050318.2019.

Sando R, Bushong E, Zhu Y, Huang M, Considine C, Phan S, Ju S, Uytiepo M, Ellisman M, Maximov A (2017) Assembly of excitatory synapses in the absence of glutamatergic neurotransmission. Neuron 94:312-321.e3.

Schätzle P, da Silva ME, Tas RP, Katrukha EA, Hu HY, Wierenga CJ, Kapitein LC, Hoogenraad CC (2018) Activity-dependent actin remodeling at the base of dendritic spines promotes microtubule entry. Curr Biol 28:2081-2093.e6.

Schindelin J, Arganda-Carreras I, Frise E, Kaynig V, Longair M, Pietzsch T, Preibisch S, Rueden C, Saalfeld S, Schmid B, Tinevez JY, White DJ, Hartenstein V, Eliceiri K, Tomancak P, Cardona A (2012) Fiji: an open-source platform for biological-image analysis. Nat Methods 9:676-682.

Shao Q, Lindstrom K, Shi R, Kelly J, Schroeder A, Juusola J, Levine KL, Esseltine JL, Penuela S, Jackson MF, Laird DW (2016) A germline variant in the PANX1 gene has reduced channel function and is associated with multisystem dysfunction. I Biol Chem 291:12432-12443.

Sigler A, Oh WC, Imig C, Altas B, Kawabe H, Cooper BH, Kwon H-B, Rhee J-S, Brose N (2017) Formation and maintenance of functional spines in the absence of presynaptic glutamate release. Neuron 94:304-311.e4. 
Spence EF, Kanak DJ, Carlson BR, Soderling SH (2016) The Arp2/3 complex is essential for distinct stages of spine synapse maturation, including synapse unsilencing. J Neurosci 36:9696-9709.

Südhof TC (2018) Towards an understanding of synapse formation. Neuron 100:276-293.

Swayne LA, Boyce AKJ (2017) Regulation of pannexin 1 surface expression by extracellular ATP: potential implications for nervous system function in health and disease. Front Cell Neurosci 11:230.

Vanden Berghe T, Hulpiau P, Martens L, Vandenbroucke RE, Van Wonterghem E, Perry SW, Bruggeman I, Divert T, Choi SM, Vuylsteke M, Shestopalov VI, Libert C, Vandenabeele P (2015) Passenger mutations confound interpretation of all genetically modified congenic mice. Immunity 43:200-209.

Vogt A, Hormuzdi SG, Monyer H (2005) Pannexin1 and Pannexin2 expression in the developing and mature rat brain. Brain Res Mol Brain Res 141:113-120.

Wicki-Stordeur LE, Swayne LA (2013) Panx1 regulates neural stem and progenitor cell behaviours associated with cytoskeletal dynamics and interacts with multiple cytoskeletal elements. Cell Commun Signal 11:62.

Xu X, Wicki-Stordeur LE, Sanchez-Arias JC, Liu M, Weaver MS, Choi CSW, Swayne LA (2018) Probenecid disrupts a novel pannexin 1collapsin response mediator protein 2 interaction and increases microtubule stability. Front Cell Neurosci 12:124.
Yang D, He Y, Muñoz-Planillo R, Liu Q, Núñez G (2015) Caspase11 requires the pannexin-1 channel and the purinergic $P 2 X 7$ pore to mediate pyroptosis and endotoxic shock. Immunity 43:923-932.

Yin J, Yuan Q (2015) Structural homeostasis in the nervous system: a balancing act for wiring plasticity and stability. Front Cell Neurosci 8:439.

Zack GW, Rogers WE, Latt SA (1977) Automatic measurement of sister chromatid exchange frequency. J Histochem Cytochem 25:741-753.

Zhang H, Kang E, Wang Y, Yang C, Yu H, Wang Q, Chen Z, Zhang C, Christian KM, Song H, Ming G, Xu Z (2016) Brain-specific Crmp 2 deletion leads to neuronal development deficits and behavioural impairments in mice. Nat Commun 7:11773.

Ziv NE, Smith SJ (1996) Evidence for a role of dendritic filopodia in synaptogenesis and spine formation. Neuron 17:91-102.

Zoidl G, Petrasch-Parwez E, Ray A, Meier C, Bunse S, Habbes H-W, Dahl G, Dermietzel R (2007) Localization of the pannexin1 protein at postsynaptic sites in the cerebral cortex and hippocampus. Neuroscience 146:9-16.

Zuo Y, Lin A, Chang P, Gan WB (2005) Development of long-term dendritic spine stability in diverse regions of cerebral cortex. Neuron 46:181-189. 